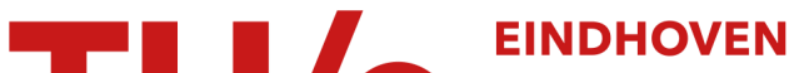 UNIVERSITY OF TECHNOLOGY
}

\section{Effect of viscosity on droplet-droplet collisional interaction}

\section{Citation for published version (APA):}

Finotello, G., Padding, J. T., Deen, N. G., Jongsma, A., Innings, F., \& Kuipers, J. A. M. (2017). Effect of viscosity on droplet-droplet collisional interaction. Physics of Fluids, 29(6), [067102]. https://doi.org/10.1063/1.4984081

DOI:

10.1063/1.4984081

Document status and date:

Published: 06/06/2017

\section{Document Version:}

Publisher's PDF, also known as Version of Record (includes final page, issue and volume numbers)

\section{Please check the document version of this publication:}

- A submitted manuscript is the version of the article upon submission and before peer-review. There can be important differences between the submitted version and the official published version of record. People interested in the research are advised to contact the author for the final version of the publication, or visit the $\mathrm{DOI}$ to the publisher's website.

- The final author version and the galley proof are versions of the publication after peer review.

- The final published version features the final layout of the paper including the volume, issue and page numbers.

Link to publication

\section{General rights}

Copyright and moral rights for the publications made accessible in the public portal are retained by the authors and/or other copyright owners and it is a condition of accessing publications that users recognise and abide by the legal requirements associated with these rights.

- Users may download and print one copy of any publication from the public portal for the purpose of private study or research.

- You may not further distribute the material or use it for any profit-making activity or commercial gain

- You may freely distribute the URL identifying the publication in the public portal.

If the publication is distributed under the terms of Article $25 \mathrm{fa}$ of the Dutch Copyright Act, indicated by the "Taverne" license above, please follow below link for the End User Agreement:

www.tue.nl/taverne

\section{Take down policy}

If you believe that this document breaches copyright please contact us at:

openaccess@tue.nl

providing details and we will investigate your claim. 


\section{Effect of viscosity on droplet-droplet collisional interaction}

Giulia Finotello, Johan T. Padding, Niels G. Deen, Alfred Jongsma, Fredrik Innings, and J. A. M. Kuipers

Citation: Physics of Fluids 29, 067102 (2017); doi: 10.1063/1.4984081

View online: http://dx.doi.org/10.1063/1.4984081

View Table of Contents: http://aip.scitation.org/toc/phf/29/6

Published by the American Institute of Physics

\section{Articles you may be interested in}

Capillary breakup of armored liquid filaments

Physics of Fluids 29, 062103 (2017); 10.1063/1.4984836

Droplets passing through a soap film

Physics of Fluids 29, 062110 (2017); 10.1063/1.4986798

Collisions of viscous droplets

Physics Today 70, 23 (2017); 10.1063/PT.3.3652

Droplet impact onto a solid sphere: Effect of wettability and impact velocity

Physics of Fluids 29, 062111 (2017); 10.1063/1.4990088

Numerical research on the dynamic characteristics of a droplet impacting a hydrophobic tube Physics of Fluids 29, 062105 (2017); 10.1063/1.4986526

Clippers, yachts, and the false promise of the wave line

Physics Today 70, 52 (2017); 10.1063/PT.3.3627

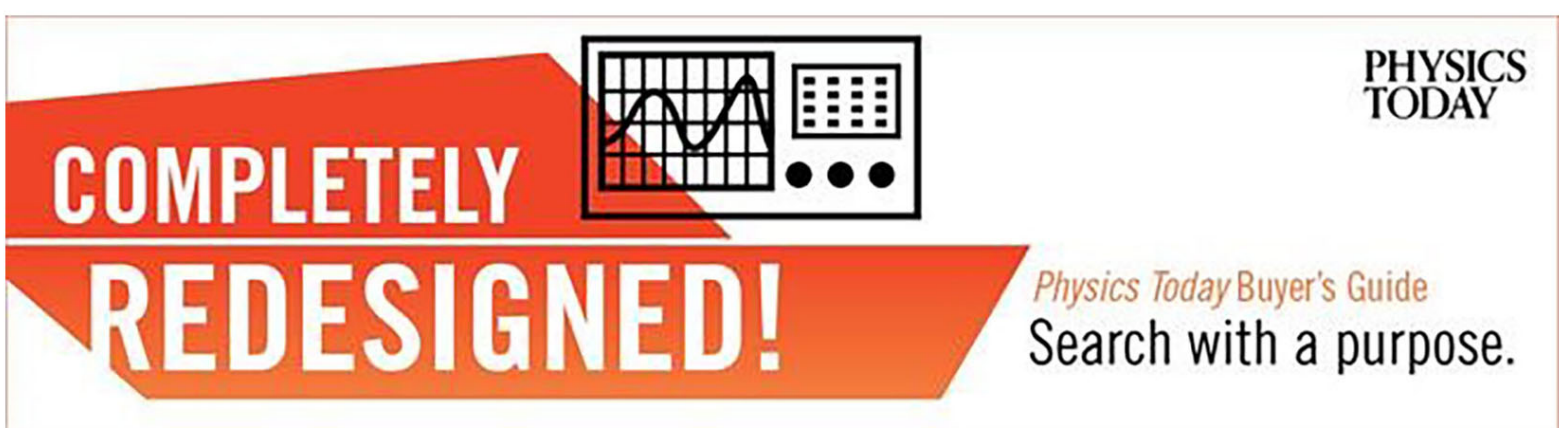




\title{
Effect of viscosity on droplet-droplet collisional interaction
}

\author{
and J. A. M. Kuipers ${ }^{1}$ \\ ${ }^{1}$ Multiphase Reactor Group, Department of Chemical Engineering and Chemistry, \\ Eindhoven University of Technology, Eindhoven, The Netherlands \\ ${ }^{2}$ Intensified Reaction and Separation Systems, Department of Process and Energy, \\ Delft University of Technology, Delft, The Netherlands \\ ${ }^{3}$ Multiphase and Reactive Flows Group, Department of Mechanical Engineering, \\ Eindhoven University of Technology, Eindhoven, The Netherlands \\ ${ }^{4}$ Tetra Pak CPS, Heerenveen, The Netherlands
}

Giulia Finotello, ${ }^{1, a)}$ Johan T. Padding, ${ }^{2}$ Niels G. Deen, ${ }^{3}$ Alfred Jongsma, ${ }^{4}$ Fredrik Innings, ${ }^{4}$

(Received 19 December 2016; accepted 11 May 2017; published online 6 June 2017)

\begin{abstract}
A complete knowledge of the effect of droplet viscosity on droplet-droplet collision outcomes is essential for industrial processes such as spray drying. When droplets with dispersed solids are dried, the apparent viscosity of the dispersed phase increases by many orders of magnitude, which drastically changes the outcome of a droplet-droplet collision. However, the effect of viscosity on the droplet collision regime boundaries demarcating coalescence and reflexive and stretching separation is still not entirely understood and a general model for collision outcome boundaries is not available. In this work, the effect of viscosity on the droplet-droplet collision outcome is studied using direct numerical simulations employing the volume of fluid method. The role of viscous energy dissipation is analysed in collisions of droplets with different sizes and different physical properties. From the simulations results, a general phenomenological model depending on the capillary number $(\mathrm{Ca}$, accounting for viscosity), the impact parameter (B), the Weber number (We), and the size ratio $(\Delta)$ is proposed. Published by AIP Publishing. [http://dx.doi.org/10.1063/1.4984081]
\end{abstract}

\section{INTRODUCTION}

The dynamics of droplet-droplet collisions has been the subject of numerous numerical, ${ }^{1-3}$ and experimental, ${ }^{4-7}$ investigations because of its complexity as a fluid dynamics phenomenon as well as its relevance for various applications in meteorology and industrial processes. Examples of these applications are the prediction of the behaviour of atmospheric raindrops, pollution tracking, liquid-liquid extraction, spray combustion, and spray drying. In particular, spray drying is an operation used in many process industries to produce powders from suspensions containing solid particles. The suspension is atomized to produce fine droplets that are dried in a hot air stream. The process involves complex, multiphase, multi-scale transport phenomena with reciprocal interactions between drying air, droplets, and solid or partially solidified particles. Furthermore, each phase is not a pure substance but is a mixture of several components. The quality of the final product is significantly affected by coalescence, break-up, and agglomeration processes prevailing during spray drying. In order to optimize the powder morphology towards the desired characteristics, it is important to have a detailed knowledge of the phenomena taking place at the individual droplet scale. Previous modelling studies on droplet collisions employed water as the medium of investigation so an explicit viscosity dependence of the droplet collision outcome was not addressed. It is our aim in this work to formulate a general model, enabling

${ }^{\text {a) Electronic mail: G.Finotello@tue.nl }}$ us to describe and predict the regime boundaries between collision outcomes. Figure 1 illustrates three examples of collision outcomes predicted by numerical simulations of this work. The first case (left part of the figure) is a reflexive separation with the formation of one satellite resulting from two droplets impacting on the same axis. The second case (central part of the figure) is an example of coalescence. The last case (right side of the figure) occurs at a high impact velocity after stretching and consequent separation with three satellites of different sizes. Each frame is associated with a non-dimensional time scaled by the initial droplet diameter and relative velocity. The bouncing regime is not represented because it is not a part of this study. This is related to limitations of the volume of fluid (VOF) method which will be discussed in detail later.

In recent years, there has been growing interest to use Computational Fluid Dynamics (CFD) for exploring phenomena of droplet interactions. A front-tracking (FT) method was used by Unverdi and Tryggvason ${ }^{8}$ and extended by Nobari and Tryggvason ${ }^{9}$ to track bouncing, coalescence, and separation for low viscosity liquids. Pan and Suga ${ }^{1}$ successfully compared the numerical bouncing collisions with a model, based on experimental results, proposed by Estrade et al. ${ }^{10}$ These numerical simulations were performed with a level set method. Sakakibara and Inamuro ${ }^{11}$ used a lattice Boltzmann method to study different droplet size ratios and viscosities. It was found that as the inertial forces increase, the mixing rate for the smaller droplet increases while the mixing rate for the larger droplet decreases. Georjon and Reitz ${ }^{12}$ developed a model for droplet coalescence and stretching separation occurring at a 


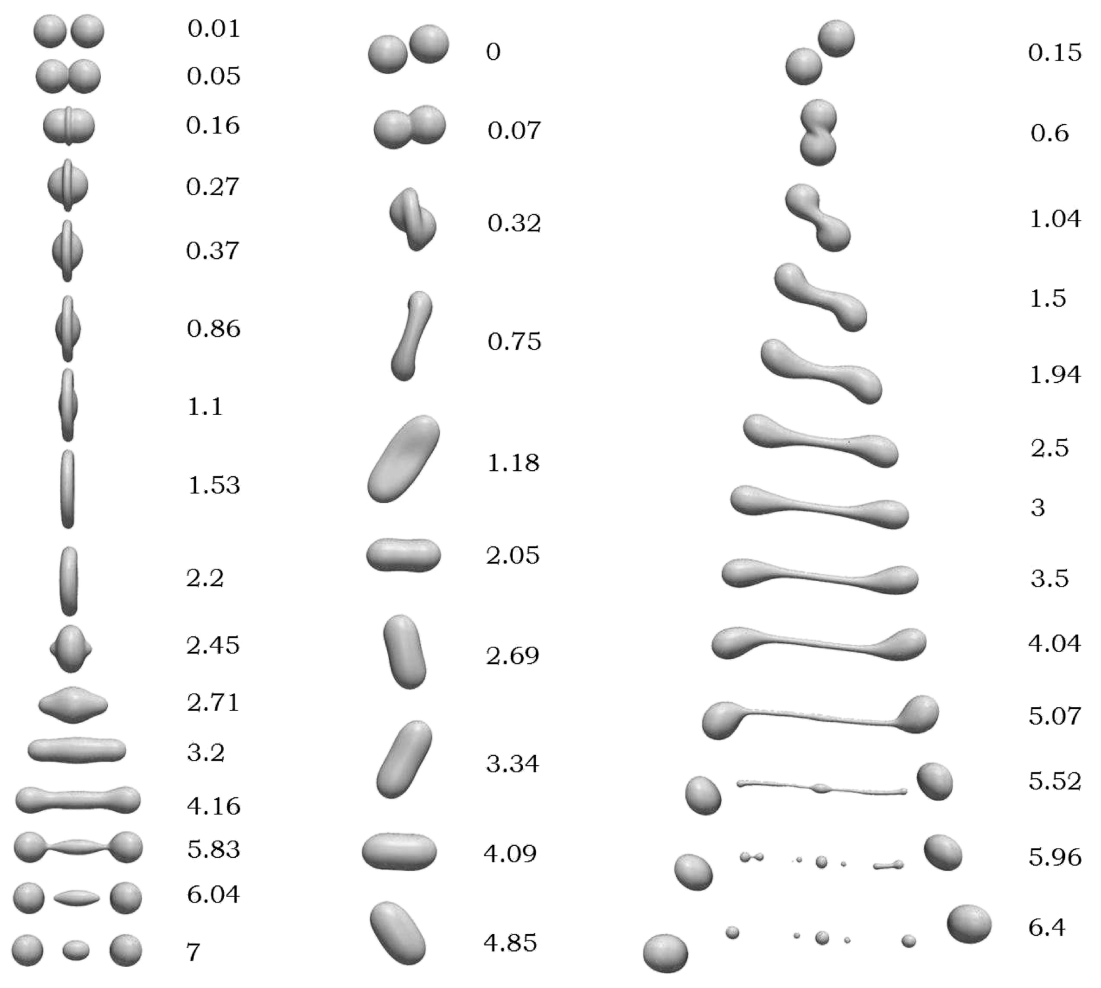

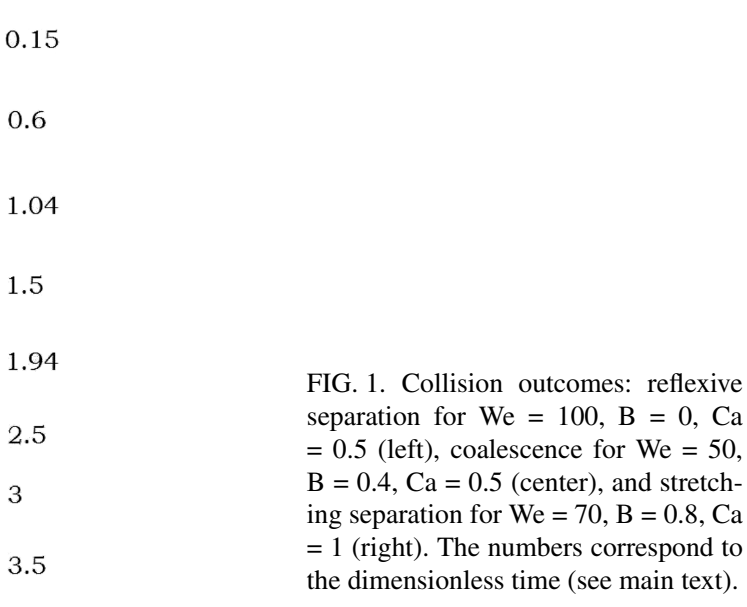

FIG. 1. Collision outcomes: reflexive separation for $\mathrm{We}=100, \mathrm{~B}=0, \mathrm{Ca}$ (left) coalescence for $\mathrm{We}=50$ ing separation for $\mathrm{We}=70, \mathrm{~B}=0.8, \mathrm{Ca}$ $=1$ (right). The numbers correspond to the dimensionless time (see main text) high We. Munnannur and Reitz ${ }^{13}$ proposed a new model for droplet collision outcomes with We numbers above 40. The collision dynamics were predicted by Monte Carlo and discrete particle methods. The spray code was adapted to track individual droplets and their collision events. In their work, Ashgriz and $\mathrm{Poo}^{6}$ models were used as a theoretical basis for the derivation of the collision outcome boundaries. For the stretching separation, they assumed that the dissipated energy is $30 \%$ of the total initial kinetic energy since the estimation was giving reasonable predictions. For the reflexive separation, $50 \%$ of the initial total kinetic energy was assumed to be dissipated. The final aim to develop a comprehensive and computationally inexpensive droplet collision model was accomplished. The volume of fluid (VOF) method was used by PassandidehFard and Roohi ${ }^{14}$ for water with unequal drop sizes. With the variation of the Reynolds ( $\mathrm{Re}$ ) number (note that all dimensionless numbers mentioned in this Introduction will be defined in Sec. II), it was found that the drop shape oscillation time and its amplitude decrease with increasing viscosity. A detailed analysis of the collision process predicted by VOF, with a focus on ligament formation and dimensions, liquid-gas interface region, and the creation of satellite droplets, is provided by Nikolopoulos, Nikas, and Bergeles ${ }^{3}$ for head-on collisions and by Nikolopoulos, Theodorakakos, and Bergeles ${ }^{15}$ for offcenter collisions. Dai and Schmidt ${ }^{16}$ conducted numerical simulations using a three dimensional moving mesh unstructured finite volume method of head-on equal size droplet collisions. They studied the effect of viscosity, finding that the dissipated energy and the maximum deformation amplitude increase with Re. Chen et al. ${ }^{17}$ performed an analysis of the energy budget for one case of reflexive separation and one case of stretching separation. The proposed model for stretching separation, based on the surface and kinetic energy at the droplet maximum deformation, does not take into account the influence of droplet viscosity. More recently, Planchette et al. ${ }^{18}$ compared experimental results with VOF simulations based and developed on a collision dynamics model by combining energy balances and Rayleigh-like criterion. The dynamic of the merged drops and consequent ligament is modelled as a liquid spring. Their study only considered the coalescence-reflexive separation boundary, i.e., only nearly head-on collisions, for a range of viscosity between 1 and $20 \mathrm{mPa}$.

Compared to numerical studies, a larger number of experimental works is devoted to droplet collisions. Brenn and Frohn ${ }^{19}$ and Ashgriz and Givi ${ }^{20}$ were among the first who studied collisions of hydrocarbon droplets showing the correlation of collision outcomes to the Weber number (We). An extensive experimental campaign on binary water droplet collisions was presented by Ashgriz and $\mathrm{Poo}^{6}$ for various impact conditions. Beyond coalescence, two separating collision regimes have been identified, reflexive and stretching separation, and theoretical models for their occurrence have been provided. Only in the work of Jiang, Umemura, and $\mathrm{Law}^{21}$ a model which explicitly includes the viscosity has been proposed. The experiments were limited to a small range of viscosities, from 0.4 to $3.5 \mathrm{mPa}$, for water and alkanes. They showed that the onset of reflexive separation increases to a higher We as the liquid viscosity to surface tension ratio increases. The model was later refined with the Ohnesorge number $(\mathrm{Oh})$ by Qian and Law. ${ }^{7}$ Furthermore Jiang, Umemura, and $\mathrm{Law}^{21}$ concluded that the extent of viscous energy dissipation occurring during the initial stage of droplet deformation is independent of the droplet viscosity. Willis and Orme ${ }^{22}$ and Willis and Orme ${ }^{23}$ conducted an experimental investigation of viscous droplet collisions in a vacuum environment to avoid aerodynamic effects during collisions. Their results showed a proportional dependency of energy dissipation on droplet viscosity, in contradiction with Jiang, Umemura, and Law. ${ }^{21}$ In the work of Brenn, Valkovska, 
and Danov ${ }^{24}$ a model for the prediction of satellite formation after the stretching separation of equal sized binary collision was developed. Experiments with propanol showed that the time to break the ligament between the two drops increases with the We and decreases for the higher impact parameter. They also found a dependency of the filament length on the impact parameter. Brenn and Kolobaric ${ }^{25}$ extended the work of Brenn, Valkovska, and Danov ${ }^{24}$ by including the effect of viscosity. The model gave good predictions for highly viscous liquids and high Wes but was not able to describe liquids such as water and alcohol. Gotaas et al. ${ }^{26}$ studied experimentally and numerically the influence of viscosity in a range from 0.9 to $50 \mathrm{mPa}$ s, analysing n-decane, mono-, di-, and tri-ethylenglycole. The analysis for model development was restricted to the boundary between coalescence and stretching separation. In general, it was observed that this boundary shifts to a higher We for fluids with higher viscosities. Reflexive separation was not identified in the experiments due to a limitation on the amplitude modulation frequency of the setup which affects the interdroplet distance. Through modification of the mutual distance between consecutive droplets, it was possible to obtain data for the onset of reflexive separation. Gotaas et al. ${ }^{26}$ confirmed that the results for the onset of reflexive separation for viscous fluids provided by Jiang, Umemura, and $\mathrm{Law}^{21}$ were not valid and a new empirical correlation was presented. In the study of Kurt, Fritsching, and Schulte, ${ }^{27}$ the collision behaviour for pure liquids and suspensions was explored. The number of satellite droplets was found to increase with viscosity. The opposite behaviour was noticed for fluids with solid particles. Furthermore an instability in the mono-dispersed droplet chain was identified for suspensions. An extensive experimental investigation on the effect of viscosity was carried out by Kuschel and Sommerfeld. ${ }^{28}$ For the coalescence-stretching separation boundary, the model of Ashgriz and Poo $^{6}$ was considered inadequate by Kuschel and Sommerfeld ${ }^{28}$ for capillary numbers (Cas) greater than 0.577 . To consider a large viscosity range, they applied a combination of Ashgriz and $\mathrm{Poo}^{6}$ and Jiang, Umemura, and Law ${ }^{21}$ models. The boundary of coalescence-reflexive separation was observed only for small solid mass fractions. Sommerfeld and Kuschel ${ }^{29}$ experimentally extended the previous work of Kuschel and Sommerfeld ${ }^{28}$ considering different alcohols and an oil. The derived models are, also in this case, a combination of Ashgriz and Poo $^{6}$ and Jiang, Umemura, and Law ${ }^{21}$ models. Recently Krishnan and Loth ${ }^{30}$ collected all the available experimental data expressing empirical models for all the collision outcome boundaries. They indicated the limitations of these models due to experimental uncertainty and important changes in collision conditions between different experiments.

Numerical investigations have the advantage of capturing all details of the internal motion of droplets during collision, which is extremely difficult in small scale experiments. In this paper, viscous dissipation energy, prevailing during impact evolution, is numerically analysed.

Many studies focussed on collision boundaries based on energy balances but a quantitative evaluation of the different energy contributions for different collision outcomes is still not available. In this work, we carry out an extensive energy balance analysis for a large number of collisions including coalescence, reflexive, and stretching separation to be able to quantitatively describe the influence of the viscous dissipation energy. The effect of viscous dissipation is then translated to boundary models depending on non-dimensional parameters.

\section{THEORY}

\section{A. Droplet-droplet dimensionless collision parameters}

To characterize the droplet-droplet collision outcome, mostly collision maps are being used, where the nondimensional impact parameter is plotted versus the collision Weber number. From previous studies by Qian and $\mathrm{Law}^{7}$ and Ko and Ryou, ${ }^{31}$ it is known that such maps based only on these two dimensionless numbers are not universal but specific to the droplet substance, in particular the droplet viscosity. We therefore need to take into consideration at least one other dimensionless number that characterizes the relative importance of viscosity. The definitions of the dimensionless parameters characterizing a collision of two droplets are given below. The Weber number is the ratio between inertial forces and surface tension

$$
W e=\frac{\rho_{d} d_{s}\left|v_{r e l}^{2}\right|}{\sigma}
$$

where $\rho_{d}$ is the droplet density, $d_{s}$ is the diameter of the smallest droplet, $v_{\text {rel }}$ is the relative velocity, and $\sigma$ is the surface tension. The ratio between $d_{s}$ and the large droplet diameter $d_{l}$ is the size ratio $\Delta$. The impact parameter $\mathrm{B}$ is defined, before the moment of impact, as the distance $\mathrm{b}$ between the two droplet centres in the plane perpendicular to the relative velocity vector (Fig. 2), normalized by the average droplet diameter

$$
B=\frac{2 b}{d_{s}+d_{l}} .
$$

When $B$ is equal to 0 it is a head-on collision and when it is 1 a grazing collision.

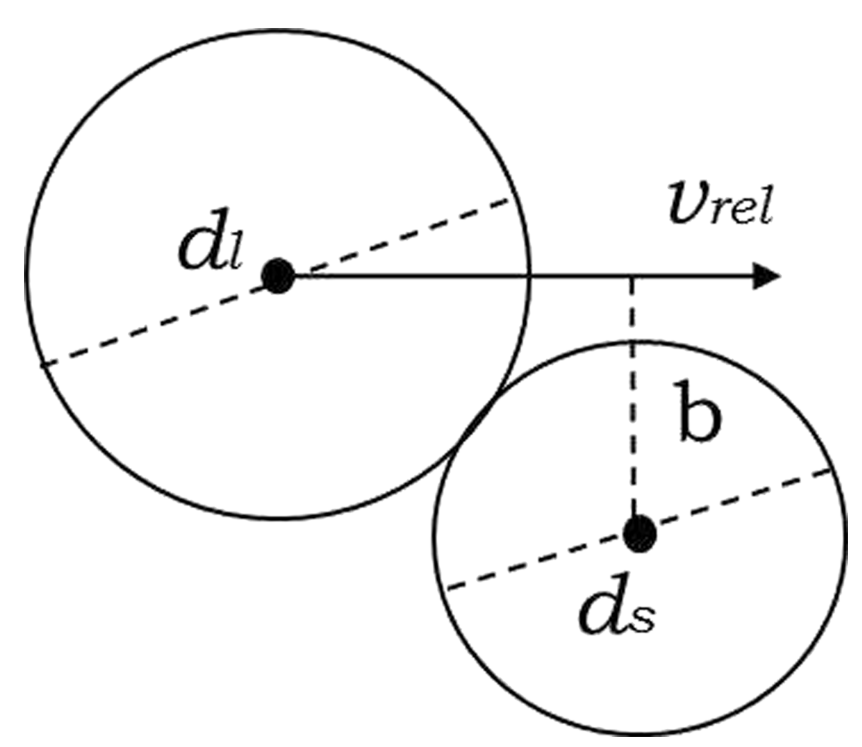

FIG. 2. Droplet collisional system geometry. 
The Ohnesorge number represents the ratio of viscous forces and the combined effect of inertial forces and surface tension

$$
O h=\frac{\mu_{d}}{\sqrt{\rho_{d} d_{s} \sigma}} .
$$

The capillary number is another non-dimensional parameter accounting for viscosity, directly indicating the ratio of viscous forces and surface tension

$$
C a=\frac{\mu_{d}\left|v_{r e l}\right|}{\sigma} .
$$

Finally, some authors use the droplet Reynolds number, defined as the ratio of the inertial and viscous forces

$$
R e=\frac{\rho_{d}\left|v_{r e l}\right| d_{s}}{\mu_{d}} .
$$

It is important to note that next to the We, only one additional dimensionless number is needed to include the effect of droplet viscosity. For example, when $\mathrm{Ca}$ is specified, Oh and Re can be calculated according to

$$
\begin{gathered}
O h=C a / \sqrt{W e}, \\
R e=W e / C a .
\end{gathered}
$$

\section{B. Current phenomenological models for regime boundaries}

For the critical We, by demarcating the change from coalescence to reflexive separation for head-on collisions $(B=0)$, Jiang, Umemura, and $\mathrm{Law}^{21}$ proposed a direct dependency on the viscosity

$$
W e_{c}=C_{1} \frac{\mu_{d}}{\sigma}+C_{2} .
$$

Successively Qian and Law $^{7}$ modified the viscosity to surface tension ratio with the $\mathrm{Oh}$ and used two constants related to geometrical parameters and the surface tension energy of the deformed droplet. The model for the critical impact parameter demarcating the boundary between coalescence and stretching separation proposed by the same authors is

$$
B_{c}=\frac{1}{W e^{1 / 2}}\left[1+k \frac{\mu_{d}}{\sigma}\left(\frac{\rho_{d} d_{s}}{\sigma}\right)\right],
$$

where $k$ is a constant. It takes into account that a portion of the kinetic energy will dissipate because of the viscous flow inside the droplets. The stretching energy available for separating the merged droplets is less and the separation becomes more difficult. It has to be realized that Jiang, Umemura, and Law $^{21}$ models are valid only for relatively low viscous liquid. One of the problems with Eqs. (8) and (9) is that they contain constants which are not dimensionless and therefore likely to be dependent on the system under consideration. This triggered other authors to develop theories or correlations which are presented in the dimensionless form.

For low viscous liquids, Ashgriz and $\mathrm{Poo}^{6}$ proposed a criterion for the boundary between coalescence and reflexive separation according to which the effective kinetic energy should be larger than $75 \%$ of the nominal surface energy of the merged droplets. The transition for head-on collisions $(B=0)$ between these regimes occurs at $W e_{c}=19$ when $\Delta$ is 1 . More generally, for non-zero values of $B$, the model for the boundary between reflexive separation and coalescence is given by

$$
W e_{c}=3\left[7\left(1+\Delta^{3}\right)^{2 / 3}-4\left(1+\Delta^{2}\right)\right] \frac{\Delta\left(1+\Delta^{3}\right)^{2}}{\Delta^{6} \eta_{s}+\eta_{l}},
$$

where

$$
\begin{gathered}
\eta_{s}=2(1-\xi)^{2}\left(1-\xi^{2}\right)^{1 / 2}-1, \\
\eta_{l}=2(\Delta-\xi)^{2}\left(\Delta^{2}-\xi^{2}\right)^{1 / 2}-\Delta^{3}, \\
\xi=\frac{1}{2} B(1+\Delta) .
\end{gathered}
$$

For the boundary between stretching separation and coalescence, they found

$$
W e_{c}=\frac{4\left(1+\Delta^{3}\right)^{2}\left[3(1+\Delta)(1-B)\left(\Delta^{3} \phi_{s}+\phi_{l}\right)\right]^{1 / 2}}{\Delta^{2}\left[\left(1+\Delta^{3}\right)-\left(1-B^{2}\right)\left(\phi_{s}+\Delta^{3} \phi_{l}\right)\right]},
$$

where

$$
\begin{gathered}
\phi_{s}=\left\{\begin{array}{cc}
1-\frac{1}{4 \Delta^{3}}(2 \Delta-\tau)^{2}(\Delta+\tau) & h>0.5 d_{s} \\
\frac{\tau^{2}}{4 \Delta^{3}}(3 \Delta-\tau) & h<0.5 d_{s}
\end{array},\right. \\
\phi_{l}= \begin{cases}1-\frac{1}{4}(2-\tau)^{2}(1+\tau) & h>0.5 d_{l} \\
\frac{\tau^{2}}{4}(3-\tau) & h<0.5 d_{l}\end{cases} \\
h=\frac{1}{2}\left(d_{l}+d_{s}\right)(1-B), \\
\tau=(1-B)(1+\Delta) .
\end{gathered}
$$

\begin{tabular}{|c|c|c|}
\hline Collision boundary & Ashgriz and $\mathrm{Poo}^{6}$ model & Jiang, Umemura, and $\mathrm{Law}^{21}$ model \\
\hline \multirow{3}{*}{ Reflexive separation } & Viscous energy dissipation neglected the & Viscous energy dissipation included the \\
\hline & reflexive criterion: Kinetic energy $\geq 75 \%$ surface energy & We only for head-on collision $(\mathrm{B}=0)$ \\
\hline & Viscous energy dissipation neglected the & Viscous energy dissipation included the \\
\hline Stretching separation & stretching criterion: Kinetic energy $\geq$ surface energy & inertial force $=$ viscous force + surface tension \\
\hline
\end{tabular}

The main assumptions used in the derivation of the phenomenological models are summarized in Table I.

A representation of these phenomenological models for the regime boundaries is given in Fig. 3. For the Jiang, Umemura, and Law ${ }^{21}$ model, the two constants obtained experimentally and related to saccharose by Kuschel and Sommerfeld ${ }^{28}$ were used. The Ashgriz and Poo $^{6}$ models are represented for $\Delta=1$, which is also the value considered in our simulations.

TABLE I. Main assumptions of phenomenological models. 


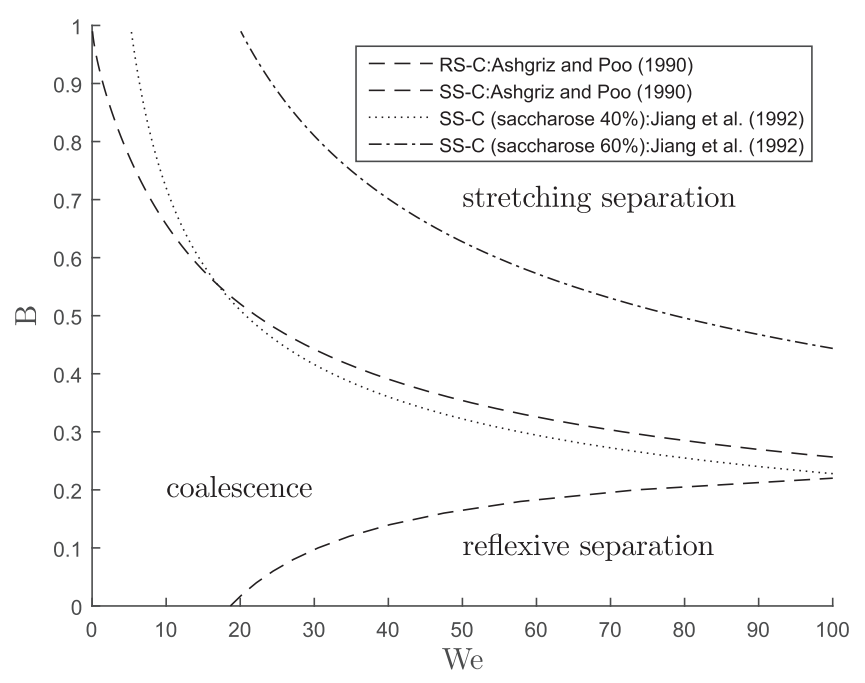

FIG. 3. Phenomenological models for reflexive separation-coalescence (RS-C) and stretching separation-coalescence (SS-C) boundaries from Ashgriz and $\mathrm{Poo}^{6}$ and Jiang, Umemura, and $\mathrm{Law}^{21}$ for the case of two saccharose solutions of different viscosity.

\section{Viscous dissipation}

The study of the viscous energy dissipation is fundamental to understand the collision dynamics of viscous droplets. Almost all theoretical models or correlations that describe the outcome for the collision boundary do not consider the dissipation because they refer to water droplets where the viscous forces are usually much less relevant than the surface tension, i.e., the capillary number is usually small. In this work, we will investigate cases where the viscous dissipation is more important. To this end, similar to Jiang, Umemura, and Law, ${ }^{21}$ we will explicitly measure the total amount of the dissipated energy $D E$, which can be related to the viscous dissipation rate $V D R$ (units $\mathrm{J} / \mathrm{s}$ ) and (local) dissipation function $\Phi$ (units $(\mathrm{J} / \mathrm{s}) / \mathrm{m}^{3}$ ) through a time integral and a spatial integral

$$
\begin{gathered}
D E(t)=\int_{0}^{t} V D R(t) d t, \\
V D R(t)=\iiint_{V_{d}} \Phi d V, \\
\Phi=\frac{1}{2} \mu_{c} \sum_{i} \sum_{j}\left(\frac{\partial u_{i}}{\partial x_{j}}+\frac{\partial u_{j}}{\partial x_{i}}\right)^{2} .
\end{gathered}
$$

The dissipation function $\Phi$ quantifies the local volumetric viscous dissipation rate. Its volume integral, the viscous energy dissipation rate $V D R$, has relatively high values from the time of droplet impact to the time the two droplets reach the maximum deformation. This time interval is not the same for all collisions because the shape and volume depend on the physical properties of the liquid, relative velocity, and impact angle. In our analysis, in principle, also viscous dissipation in the interstitial gas phase is considered because in Eq. (21) the local value of the viscosity $\mu_{c}$ is used, as explained in detail in Sec. III. Considering air as a continuous phase, we found that in all cases, the viscous dissipation inside the liquid droplets gives the dominant contribution to the overall viscous dissipation rate.

\section{NUMERICAL SIMULATIONS}

\section{A. VOF method}

The VOF method is an interface capturing technique which utilizes a color function $F$ to indicate the fractional amount of liquid in each cell. The main properties of the method are described here. For an extensive and detailed description of the numerical model, see Van Sint Annaland, Deen, and Kuipers ${ }^{32}$ and Baltussen, Kuipers, and Deen. ${ }^{33}$ Our VOF implementation is based on the continuity equation and Navier-Stokes equation for incompressible flows

$$
\begin{gathered}
\nabla \cdot u=0, \\
\frac{\partial}{\partial t}(\rho u)=-\nabla p-\nabla \cdot \rho u u+\nabla \tau+\rho g+F_{\sigma},
\end{gathered}
$$

where $F_{\sigma}$ represents the surface tension force. The velocity field is continuous even across the interface and it is calculated with a staggered grid configuration, using a projectioncorrection method. All terms in Eq. (23) are treated explicitly, except for the diffusion term which is treated semi-explicitly. The implicit part of the viscous diffusion term only depends on the Cartesian velocity component that is solved, while the remaining explicit terms are relatively small. The convective term in the Navier-Stokes equation is discretized with a second order flux-delimited Barton scheme, while for the diffusion term, a second order central difference scheme is applied. The tentative velocity field is corrected to satisfy Eq. (22). Once the velocity field is calculated, a geometrical advection is applied to the phase fraction according to the following equation:

$$
\frac{D F}{D t}=\frac{\partial F}{\partial t}+u \cdot \nabla F=0 .
$$

With the VOF method, both phases are allowed to reside in the same computational cell. Depending on the $F$ value in the neighbouring computational cells, the orientation of the gasliquid interface is reconstructed. To preserve an accurate and sharp interface, the interface is represented in planar segments according to the Piecewise Linear Interface Calculation (PLIC) algorithm of Youngs. ${ }^{34}$ The local density $\rho_{c}$ and viscosity $\mu_{c}$ are computed from the colour function $F$ as follows:

$$
\begin{gathered}
\rho_{c}=F \rho_{l}+(1-F) \rho_{g}, \\
\frac{\rho_{c}}{\mu_{c}}=F \frac{\rho_{l}}{\mu_{l}}+(1-F) \frac{\rho_{g}}{\mu_{g}},
\end{gathered}
$$

where $l$ stands for the liquid and $g$ for the gas.

The tensile force model is used in this work for the computation of the surface tension term. This approach describes the surface tension as a tensile force acting on the closed contour defined by a surface element. Each neighbouring element exerts a tensile force on a reference element because of their relative orientation. A detailed description of the model is provided by Tryggvason et al. ${ }^{35}$ within the framework of the Front Tracking (FT) method, with modifications in the implementation for the VOF method as reported by Baltussen, Kuipers, and Deen. ${ }^{33}$

A pressure jump correction based on the theory of Dijkhuizen et al. ${ }^{36}$ is implemented to avoid instabilities due to 
pressure discontinuity in the gas-solid interface. The calculation of the pressure jump at the interface is coupled with the surface tension by the following equation:

$$
[p I-\tau] \cdot n=F_{\sigma},
$$

where $I$ is the unit tensor and $\mathrm{n}$ is the interface normal. The viscous stress force components in the normal direction can be neglected and Eq. (27) is reduced to

$$
[p]=\frac{\sum_{s} F_{\sigma, m} \cdot n}{\sum_{s} S_{m}},
$$

where $F_{\sigma, m}$ is the tensile force that the neighbouring elements exert on the element $m$ and $S_{m}$ is the surface area of the element $m$.

The method is suitable to simulate coalescence and breakup with no limitation in the formation of satellites. However, the bouncing regime cannot be physically predicted. VOF models exhibit automatic coalescence when surfaces from two elements are in close proximity because the interface is reconstructed using data from the fixed grid. As a consequence, a proper prediction of the delay in the rupture of the drainage gaseous film between two droplets is not possible. In Nobari, Jan, and Tryggvason ${ }^{2}$ and Pan, Law, and Zhou, ${ }^{37}$ the rupture of the interdrop film was prescribed artificially on the basis of critical droplet shape deformation or experimental observations. In the work of MacKay and Mason, ${ }^{38}$ a critical gas film thickness of $10 \mathrm{~nm}$ was enforced as a coalescence criterion. A multiple marker method with a coupled level set volume of the fluid method was applied by Kwakkel, Breugem, and Boersma ${ }^{39}$ to prevent numerical coalescence when two droplets have an interface gap of one grid cell or less. Whichever method is used, it is usually grid-size dependent and very sensitive to the accuracy of the assumed film drainage model. A change of the gas properties, in which collision events occur, leads to changes in the bouncing probability. It was observed by Krishnan and $\operatorname{Loth}^{30}$ that an increase in the gas density stabilizes the interdrop layer with a consequent increase in the bouncing probability. Fortunately, it was also affirmed that the boundaries between coalescence and both types of separation are insensitive to the surrounding gas properties. We therefore apply (and limit) the current VOF method to study the influence of viscous dissipation on the transition from coalescence to (reflexive or stretching) separation.

\section{B. Simulation settings}

A total number of 116 binary equal-sized drop collisions have been simulated in the present work with a variation of the We between 20 and 100 and B between 0 and 0.8 . Wes below 20 and impact parameters above 0.8 are excluded a priori because a bouncing collision is expected under these conditions. Three values of $\mathrm{Ca}, 0.1,0.5$ and 1 , are considered in this investigation. The lowest value of the $\mathrm{Ca}$ is important to confirm the reflexive separation regime which occurs for relatively low viscosity liquids such as water. Table II contains the properties of the fluids used in the present simulations. Our results will apply more generally to any viscous liquid if we express the results in terms of dimensionless groups
TABLE II. Physical properties of the fluids used in this work.

\begin{tabular}{lccc}
\hline \hline Fluid & $\rho\left(\mathrm{kg} / \mathrm{m}^{3}\right)$ & $\mu(\mathrm{mPa} \mathrm{s})$ & $\sigma(\mathrm{mN} / \mathrm{m})$ \\
\hline Air & 1.25 & $18 \times 10^{-3}$ & \\
Saccharose $40 \%$ & 1177 & 6 & 75 \\
Saccharose $60 \%$ & 1287 & 57 & 77 \\
\hline \hline
\end{tabular}

(e.g., B, We, Ca) and dimensionless numerical quantities (e.g., number of grid cells per droplet diameter and the Courant number).

For the simulations, free slip boundaries are set on all six faces of the cubic computational domain. In order to contain the deformed liquid droplets during the entire collision process, the size of the computational domain is determined according to the value of the Weber number and the impact parameter. For head-on and near head-on collisions, a small domain size, with a minimum of 3 droplet diameter $d$, was sufficient. For higher B, the elongation of the drops stretching apart from each other and the formation of multiple satellites require an extension to a maximum of $7.5 \mathrm{~d}$. Initially, the two droplets are positioned near the center of the domain along the body diagonal. Depending on the impact parameter, the two centres are shifted above and below the diagonal. The initial gap between the droplets is 0.4 or 0.5 droplet radius. At the beginning of the simulation, a uniform velocity is imposed on each of the two liquid droplets in the diagonal direction while the surrounding air is quiescent. The velocities are set in such a way that relative collision velocities of $1-12 \mathrm{~m} / \mathrm{s}$ are used. The initial condition of the uniform velocity of the droplets and zero initial velocity of the gas will lead to a sudden acceleration of the gas, which in turn may result in unphysical transient in the droplet surfaces. Fortunately, since the liquid/gas density ratio is equal or higher than 4800 , the influence of the gas phase acceleration on the droplet motion can be neglected. A Cartesian uniform mesh system is used for all the present simulations. For all cases, the droplet diameter is resolved by 40 grid points. In the work of Padding et al., ${ }^{40}$ a validation case for a separation case with the formation of one satellite droplet is shown for 45 grid points in the droplet diameter. These simulations, which use the same methodology as in the current work, were compared to experiments from Qian and $\mathrm{Law}^{7}$ and simulations by Pan and Suga. ${ }^{1}$ We opt for a resolution of $d_{p} / \Delta x=40$ for all our simulations as a compromise between a good resolution, leading to reliable and consistent results, and the very demanding computational time. Because a large range of Wes and Cas are considered, the droplet diameters are in a wide range from $20 \mu \mathrm{m}$ to $2 \mathrm{~mm}$, corresponding to a grid size range of $0.5 \mu \mathrm{m}-50 \mu \mathrm{m}$. The grid resolution was chosen sufficiently small to have the grid-based Peclet number $|u| \Delta x / v$, with $v$ the kinematic viscosity of the liquid, smaller than 1.5 (must be smaller that 2 for stability). Moreover, the flow solver time step $\Delta t$ is chosen such that the Courant-Friedrichs-Lewy (CFL) number $|u| \Delta t / \Delta x$ is typically smaller than 0.2 . We have performed a grid dependency study for the extreme cases (largest and smallest droplets) and confirmed that the velocity and energy evolution after impact change by less than $10 \%$ when increasing the grid resolution by a factor of 1.5 in each direction. 
For the serial simulations, we used a Workstation Z420 and the Cartesius cluster SURF Cooperative (Amsterdam). The simulations with coalescence as the collision outcome had a duration between 2 and 3 weeks. The separation cases had a duration between 1 and 4 months, depending on the formation of the ligament and its breakup.

\section{RESULTS}

\section{A. Energy analysis}

We anticipated that the energy dissipation in a collision attains high values from the time of the droplet impact to the time when the two droplets reach the maximum deformation where this interval depends on the physical properties of the liquid, relative impact velocity, and impact angle. However, we observed that the initial merging of the two droplets is not the only process characterized by a high energy dissipation rate. Often a second or even a third peak appeared in the viscous dissipation rate versus time. This occurred mostly for collisions at the low $\mathrm{Ca}$ (low viscosity) which are characterized by a high deformation and relatively large changes in the shape of the combined surface. For higher Cas, the viscosity is sufficiently high to oppose the transversal inertial forces that elongate the merged droplet so that a high degree of droplet deformation does not appear. Deformation due to the stretching of the merged droplets, with the formation of a first liquid bridge and consecutive break-up in satellite droplets is an observed phenomenon for all Cas but it does not lead to high values of the viscous dissipation rate.

Figure 4 shows two collision evolutions for the same We of 70 and the impact parameter $\mathrm{B}$ equal to 0.5 but a different $\mathrm{Ca}$, together with a surface contour of the viscous dissipation function $\Phi$, indicating regions of relatively high viscous dissipation (in red). For the Ca 0.1, the deformation of the evolving droplet is evident and high values of viscous dissipation result also after the initial merging process, while for the $\mathrm{Ca} 1$, the shape change is less relevant and the viscous dissipation rate does not show major multiple peaks.

The complexity in the spatial and temporal distribution of the viscous dissipation function during a droplet-droplet collision has important consequences for the validity of phenomenological models which are based on simple geometrical arguments. For example, the model of Jiang, Umemura, and Law, ${ }^{21}$ which is based on the assumption that the energy is dissipated predominantly in a lens-shaped domain after the first impact when the drops merge, does not account for the total amount of the viscous energy dissipated during the complete collision.

We have tracked the energy budget of a droplet-droplet collision from the moment the drops are separated until the moment the collision outcome is evident. From energy conservation, the sum of the initial kinetic energy $K E_{\text {init }}$ and initial surface energy $S E_{\text {init }}$ of the droplets must be equal to the amount of the kinetic, surface, and dissipated energy at a considered time

$$
T E=K E_{\text {init }}+S E_{\text {init }}=K E(t)+S E(t)+D E(t),
$$

where $T E$ is the total energy. $K E(t)$ is calculated as the integral over all cells in the domain (gas and liquid) of $\frac{1}{2} \rho v^{2}$. $S E(t)$ is calculated as $\sigma S$ where $S$ is the sum of each element surface belonging to the droplet interface. $D E(t)$ is calculated from Eq. (19).

In Fig. 5, the energy budget during collision for $\mathrm{We}=20$, $\mathrm{B}=0.7$, and $\mathrm{Ca}=0.1$ is shown. The energies are normalized by the total initial energy. The viscous dissipation rate $V D R$ is also shown to highlight the period of intense viscous dissipation during the collision. In this case, the collision outcome is coalescence. The dominant peak of the VDR curve corresponds to the configuration 1 of the first impact, shown below in Fig. 5, and consecutive merging with a sudden decrease of the surface energy $S E$. After the peak, the viscous dissipation decreases and the coalesced drop returns to a spherical shape.

The energy budget during collision for $\mathrm{We}=90, \mathrm{~B}=0.4$, and $\mathrm{Ca}=1$ is illustrated in Fig. 6. In this case, the collision outcome is stretching separation with the formation of one satellite droplet.

Note that in both cases, the loss due to viscous dissipation is almost equal to the total amount of the initial kinetic energy. It has to be confirmed that a collision can be considered completed when the residual kinetic energy is negligible. In our

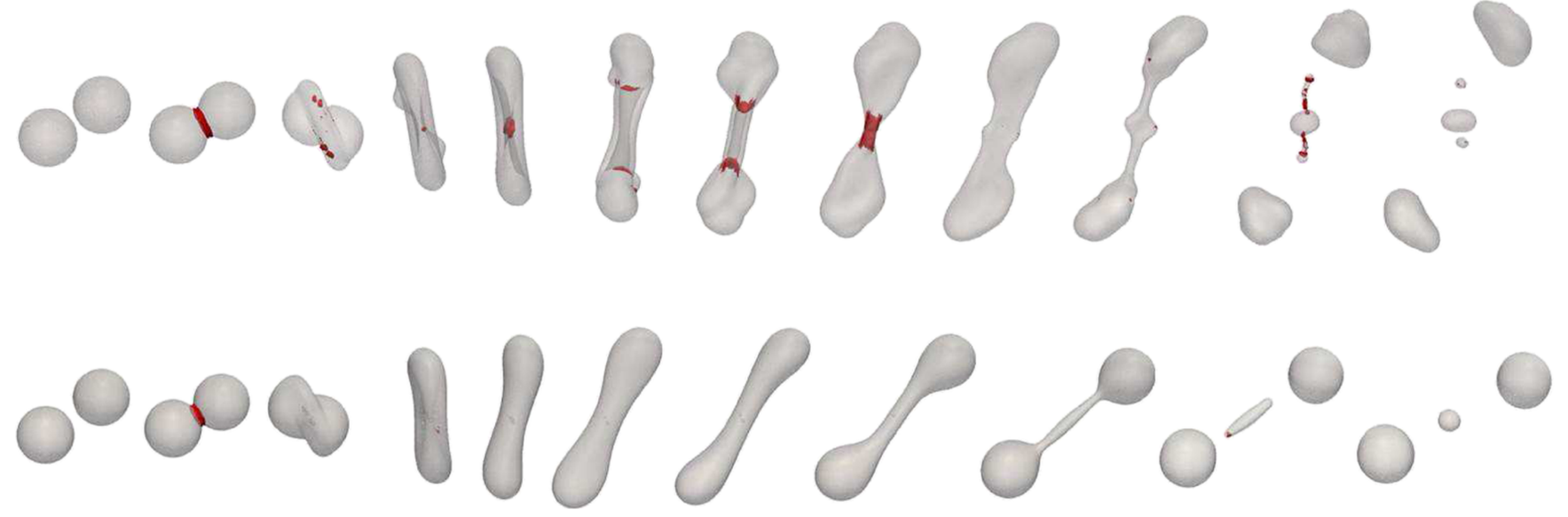

FIG. 4. Collision evolution for $\mathrm{We}=70, \mathrm{~B}=0.5$ at $\mathrm{Ca}=0.1$ (top) and $\mathrm{Ca}=1$ (bottom). Red color indicates the region of relatively high values of the viscous dissipation function $\Phi$. 
Normalized Energy budget
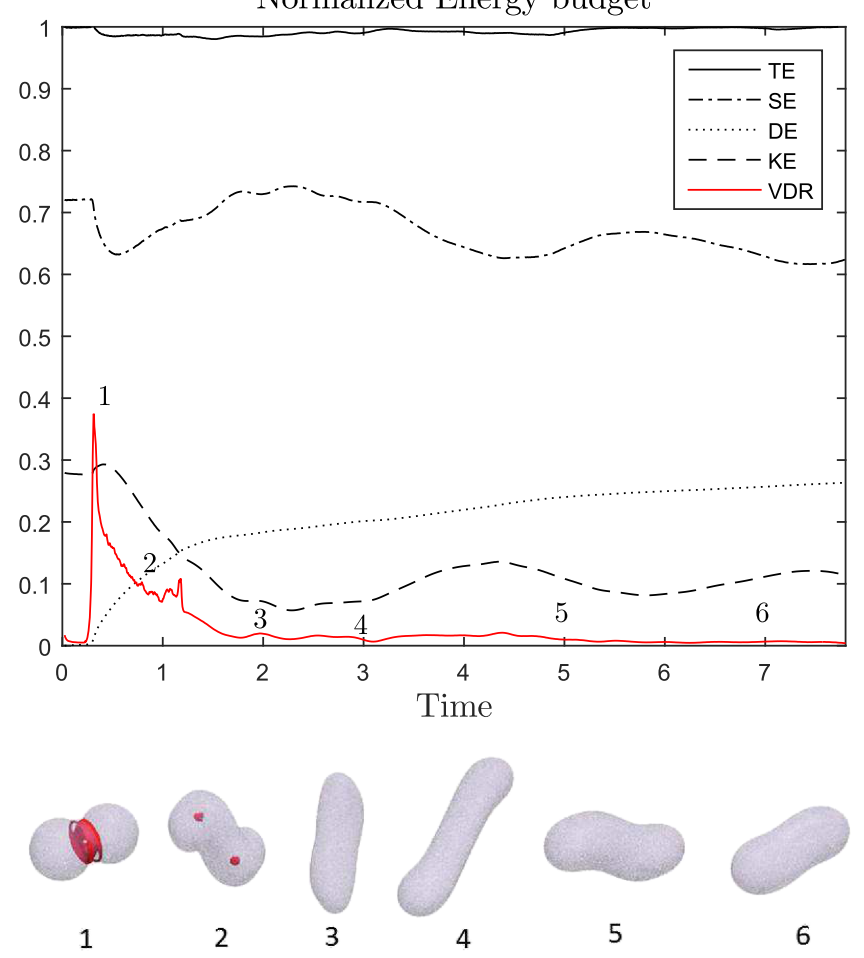

FIG. 5. Normalized energy budget of the kinetic energy KE, surface energy $\mathrm{SE}$, dissipated energy DE, and total energy TE for coalescence at $\mathrm{We}=20$, $\mathrm{B}=0.7$, and $\mathrm{Ca}=0.1$. The viscous dissipation rate VDR is given by the red line (note that an arbitrary vertical scaling was used). The time is normalized by $d / v_{\text {rel }}$.

work, the kinetic energy at the final time was always less than $10 \%$ of the total energy.

When the total normalized dissipated energy $D E$ at the final time $t_{\text {fin }}$ is plotted as a function of the impact parameter $\mathrm{B}$ for various values of We and $\mathrm{Ca}$, as displayed in Fig. 7, it is evident that the assumption that a constant fraction of the
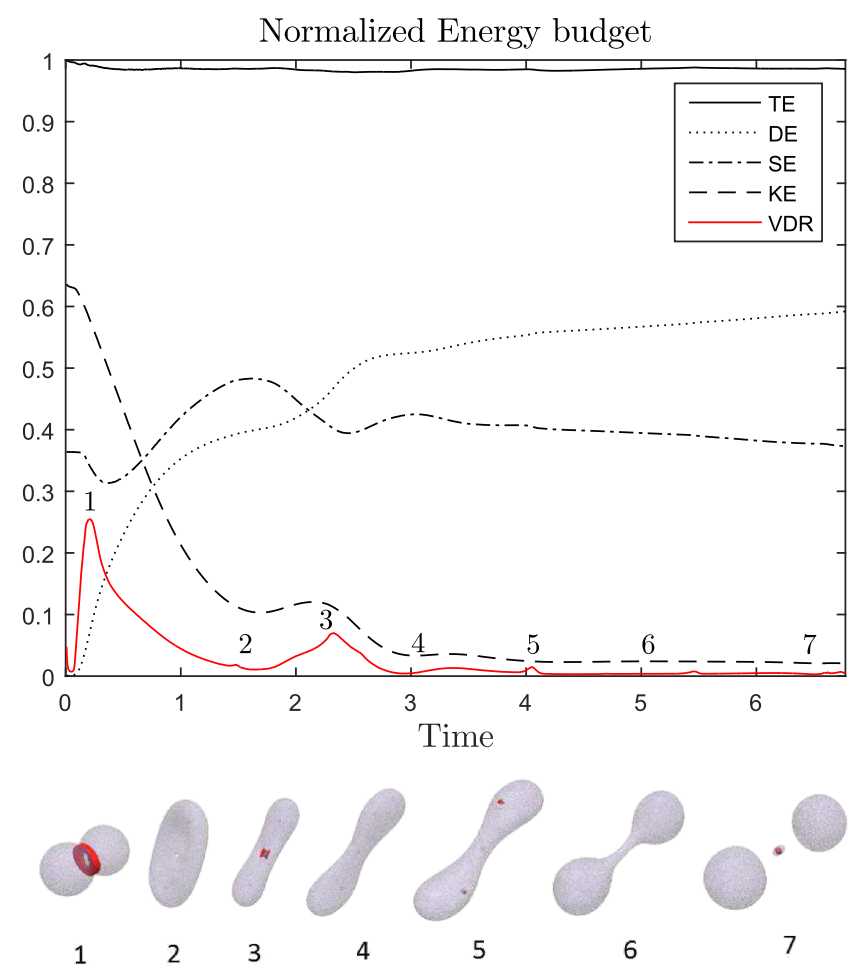

FIG. 6. Normalized energy budget of the kinetic energy KE, surface energy SE, dissipated energy DE, total energy TE for stretching separation at $\mathrm{We}=90, \mathrm{~B}=0.4$, and $\mathrm{Ca}=1$. The viscous dissipation rate VDR is given by the red line (note that an arbitrary vertical scaling was used). The time is normalized by $d / v_{\text {rel }}$.

energy is dissipated (independent of the viscosity), assumed by Jiang, Umemura, and Law, ${ }^{21}$ is too simplistic. For head-on collisions $(\mathrm{B}=0)$, the normalized $D E$ ranges from around 0.3 to 0.7 . For higher impact parameters, the portion of the droplet involved in the collision is smaller and, as a consequence, also the fraction of the dissipated energy is smaller.

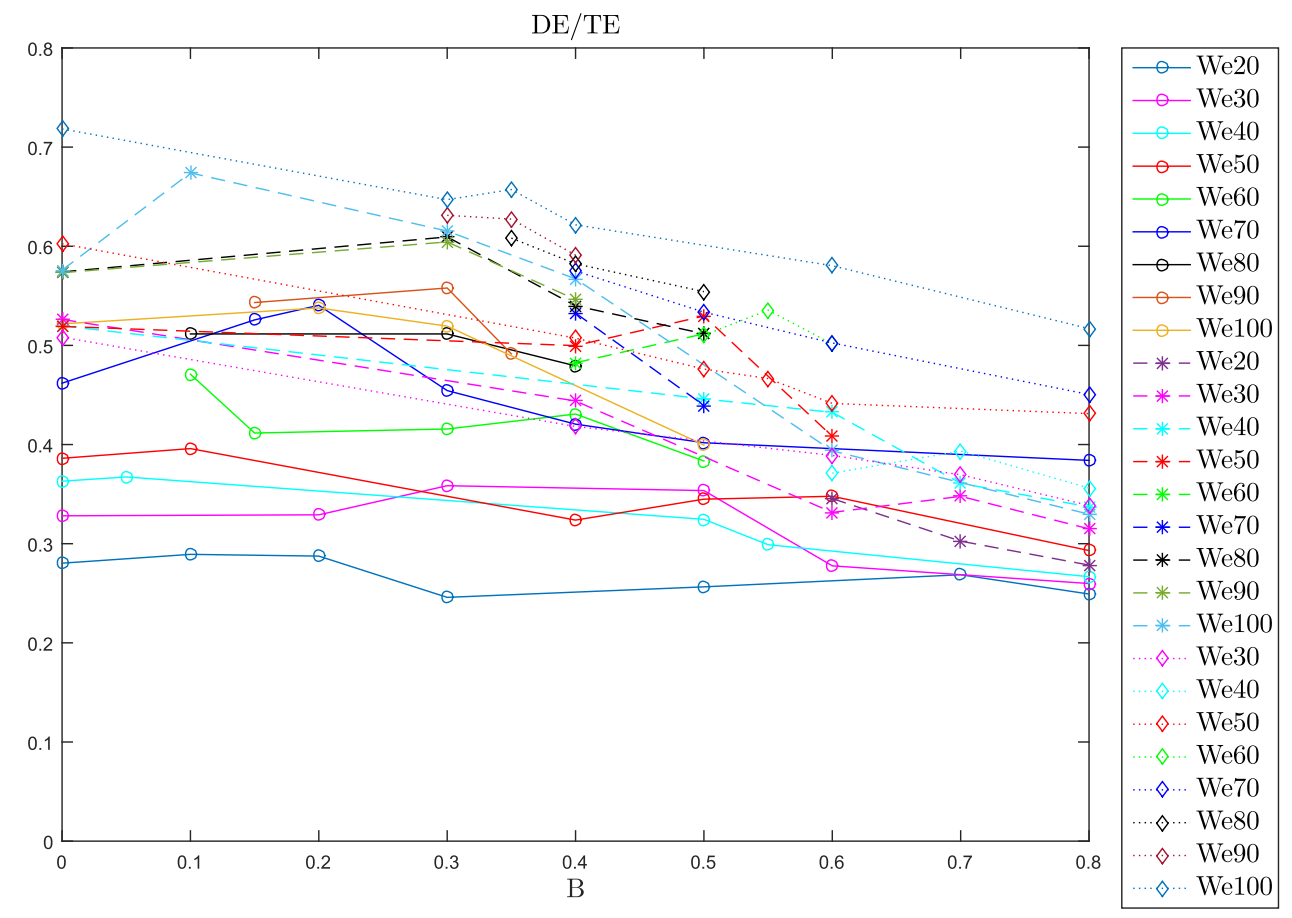

FIG. 7. The normalized viscous dissipated energy of all collision outcomes as a function of $\mathrm{B}$ for different Wes and Cas: $\mathrm{Ca} 0.1$ for continuous lines and circles, $\mathrm{Ca} 0.5$ for dashed lines and asterisks, $\mathrm{Ca} 1$ for dotted lines and diamonds. Different colors correspond to different Wes, as indicated in the legend. 
$\mathrm{DE} / \mathrm{TE}$

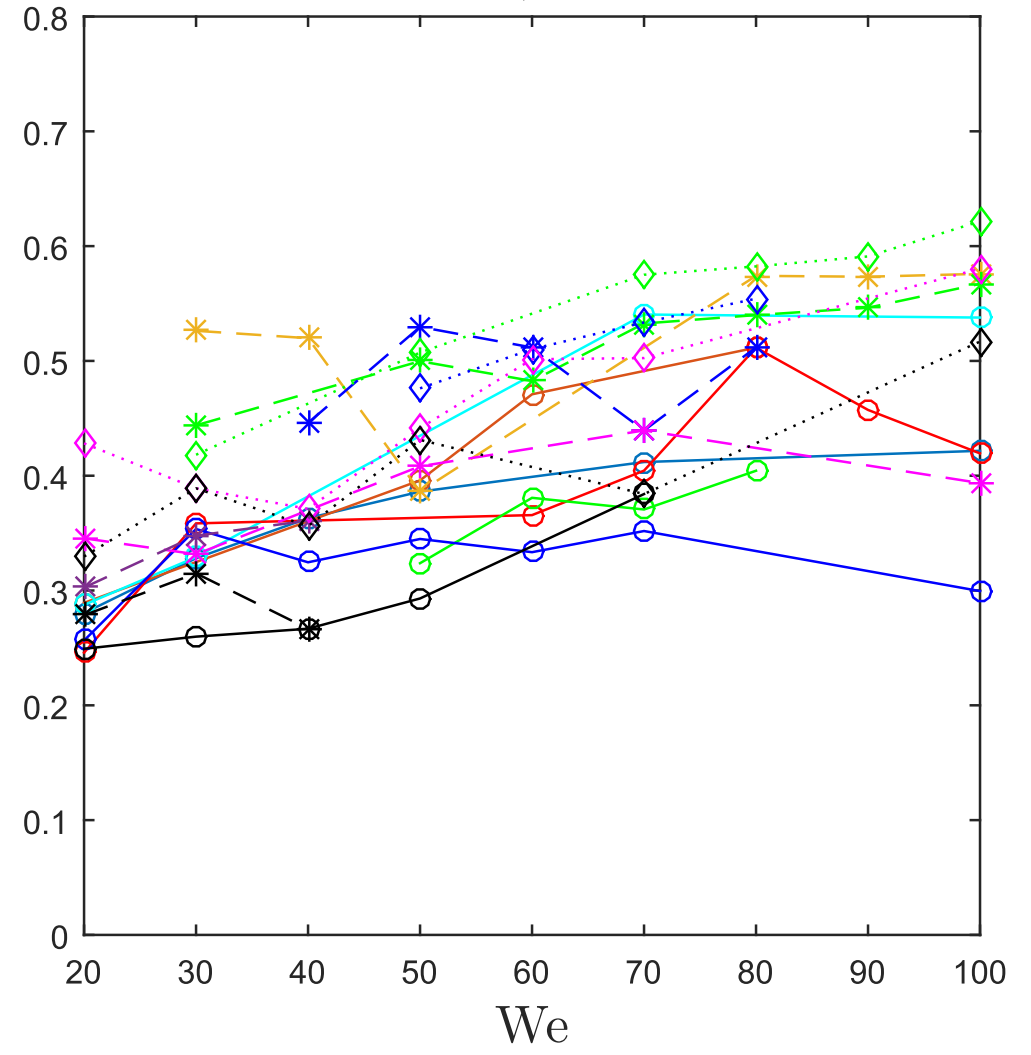

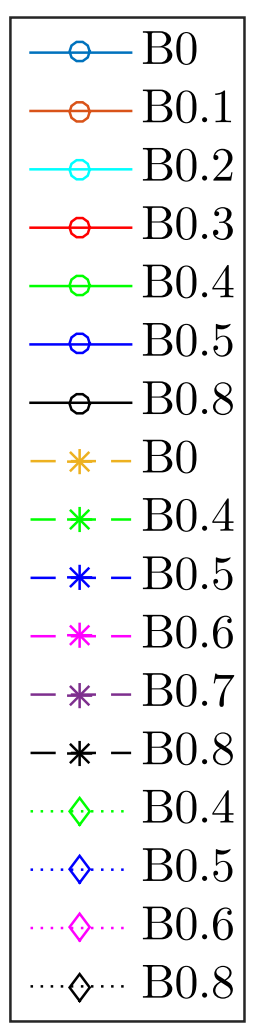

FIG. 8. The normalized viscous dissipated energy as a function of We for different Bs and Cas: $\mathrm{Ca} 0.1$ for continuous lines and circles, $\mathrm{Ca} 0.5$ for dashed lines and asterisks, $\mathrm{Ca} 1$ for dotted lines and diamonds.
In Fig. 8, we investigate the dependence of this quantity on the We, for different Cas and Bs. We find that, although there is a weak dependence on $\mathrm{We}$, the dependence on $\mathrm{Ca}$ is much stronger. We will use this observation in the development of an empirical correlation for collision outcome boundaries in Secs. IV B and IV C. Similar to Ashgriz and $\mathrm{Poo}^{6}$ we found that separate correlations need to be developed for the coalescencestretching separation boundary and the coalescence-separation boundary.

In the case of the $\mathrm{Ca} 0.1$ and We exceeding 60 , the total energy is observed to decrease over time, typically reducing by $10 \%$ over the full duration of collision. We attribute this decrease to the relatively high surface deformations occurring under these conditions, leading to small interfacial structures with high curvature. The surface forces occurring from such small structures are not well captured by the tensile force method for our current resolution of 40 cells per droplet diameter. We investigated the grid resolution dependence for one such extreme case $(\mathrm{Ca}=0.1, \mathrm{We}=70)$ and found that the total energy is better conserved with increasing resolution. However, these higher resolutions are extremely computationally demanding, so we refrained to investigate all cases at such high resolution. Fortunately, we also observed that the individual energy contributions normalized by the total energy are much less affected by grid resolution, so our results will still be valid.

\section{B. Stretching separation model}

As mentioned before, because of energy conservation the kinetic energy $K E_{\text {init }}$ and the surface energy $S E_{\text {init }}$ before collision should be equal to the amount of the kinetic, surface, and dissipated energy at any considered time. We will consider a particular time, namely, the final time $t_{f i n}$, where the collision outcome is evident, meaning that we can distinguish between coalescence, separation, and separation with the formation of satellites, and any further dissipation only acts to relax the deformed droplet shapes to spheres. For example, $t_{\text {fin }}$ in Fig. 6 is the time corresponding to the configuration number 7 where the formation of the satellite is visible. At that time, we have

$$
K E_{\text {init }}+S E_{\text {init }}=K E_{\text {fin }}+S E_{f i n}+D E\left(t_{\text {fin }}\right) .
$$

Near the boundary between coalescence and stretching separation, the amount of the new surface created will be relatively small because only a single small satellite droplet will be created. Therefore, we assume that the surface energies before collision and at time $t_{f i n}$ are almost equal, $S E_{\text {init }} \sim S E_{f i n}$. Moreover, we assume that the kinetic energy $K E_{f i n}$ is close to zero at time $t_{f i n}$. With these assumptions, the energy balance can be rewritten

$$
\frac{K E_{\text {init }}}{T E}=\frac{D E\left(t_{\text {fin }}\right)}{T E} .
$$

The right term is fitted using the measured total normalized dissipated energy as shown in Fig. 7. The fit is made under the assumption that the normalized dissipated energy depends on the $\mathrm{Ca}$ and impact parameter $\mathrm{B}$. The effect of We is neglected because we observed that the dependence on We is smaller than the dependence on B or Ca (although Fig. 8 demonstrates there is an influence especially for the $\mathrm{Ca} 1$ ). An example of the fitting procedure is illustrated in Fig. 9 for stretching separation collisions at different Cas. We assume a 
DE/TE for Stretching Separation

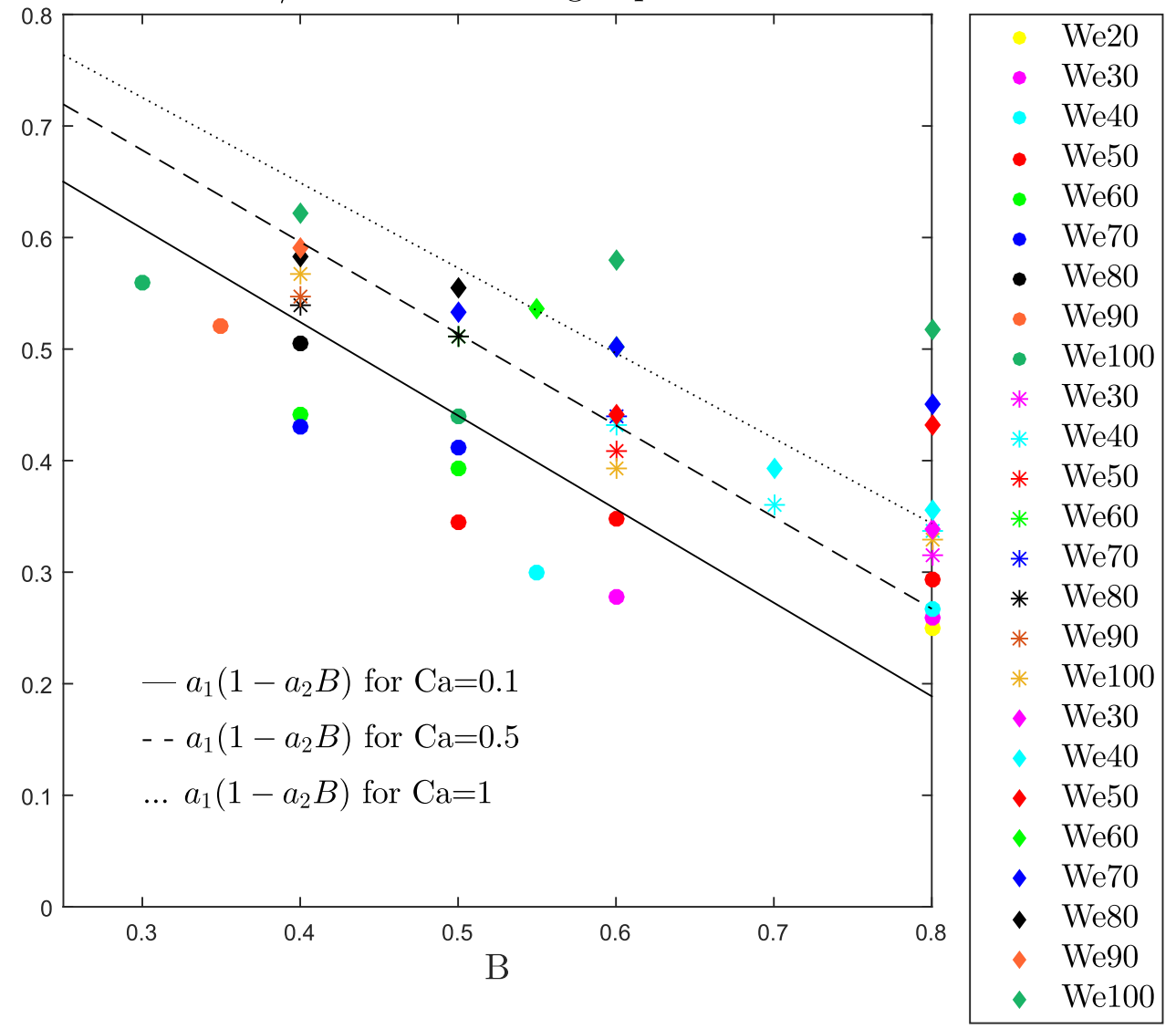

FIG. 9. The normalized viscous dissipated energy as a function of B for different Wes and Cas for stretching separation collisions and the derivation of models. Ca 0.1 for continuous line and circles, $\mathrm{Ca} 0.5$ for dashed line and asterisks, Ca 1 for dotted line and diamonds. simple linear relation between the impact parameter $B$ and the fraction of the dissipated energy $D E / T E=a_{1}\left(1-a_{2} B\right)$, where the two coefficients $a_{1}$ and $a_{2}$ of the linear relation are assumed to depend on the capillary number.

The left term of Eq. (31) can be elaborated considering pre-collision velocities of the small and large drops in the center-of-mass frame of reference, $U_{s}=\frac{v_{r e l}}{1+\Delta^{3}}$ and $U_{l}=\frac{\Delta^{3} v_{r e l}}{1+\Delta^{3}}$, where $v_{\text {rel }}$ is the pre-collisional relative velocity between the small and the large drop. Considering that the small and the large drop have a mass $\rho_{d} \pi \Delta^{3} d_{l}^{3} / 6$ and $\rho_{d} \pi d_{l}^{3} / 6$, respectively, and a surface energy $\sigma \pi \Delta^{2} d_{l}^{2}$ and $\sigma \pi d_{l}^{2}$, respectively, the large drop diameter $d_{l}$ can be simplified in favour of the We.

The model for the stretching separation is therefore expressed as a function of the We, impact parameter, size ratio, and $\mathrm{Ca}$

$$
\begin{gathered}
\frac{\frac{W e \Delta^{2}}{12\left(1+\Delta^{3}\right)\left(1+\Delta^{2}\right)}}{\frac{W e \Delta^{2}}{12\left(1+\Delta^{3}\right)\left(1+\Delta^{2}\right)}+1}=a_{1}\left(1-a_{2} B\right), \\
a_{1}=\frac{21}{22}\left(C a^{1 / 22}\right), \\
a_{2}=\frac{1}{1+\frac{1}{4} C a} .
\end{gathered}
$$

The coefficients $a_{1}$ and $a_{2}$ in Eqs. (33) and (34) were obtained empirically by fitting the observed dependence on $\mathrm{Ca}$.

\section{Reflexive separation model}

In the case of head-on collisions, Jiang, Umemura, and Law $^{21}$ empirically determined that approximately $50 \%$ of the initial kinetic energy is dissipated for water and hydrocarbon droplets collisions. On the other hand, Qian and $\mathrm{Law}^{7}$ recognized that the initial kinetic energy has to overcome the viscous dissipation from the time of coalescence until the separation of the ligament so that the initial kinetic energy lost in the deformation processes is more than $50 \%$. The losses predominantly arise from the time when drops impinge head-on and form a disk, the period when the disk contracts recovering a spherical shape, and during the last stretching in a cylinder which forms an elongating ligament (which eventually breaks). The theory was developed only for head-on collisions, identifying the boundary between coalescence and reflexive separation at $\mathrm{B}=0$. The model by Jiang, Umemura, and $\mathrm{Law}^{21}$ for the onset was reformulated as a function of the Oh, a geometrical parameter and a parameter correlated to the variation of surface tension due to deformation. The total number of results related to the reflexive separation regime is not sufficient to derive a model based on the extent of viscous energy dissipation. For this reason, the model for the boundary between coalescence and reflexive separation is expressed as a variation of the Ashgriz and $\mathrm{Poo}^{6}$ reflexive separation model, with the inclusion of the effects of viscous dissipation.

Two drops after collision will be in a state of combined mass with no translational kinetic energy but only surface energy and internal kinetic energy. Ashgriz and Poo $^{6}$ analysed reflexive separation introducing the concept of the effective reflexive energy $K_{r}^{0}$,

$$
K_{r}^{0}=K_{e}+K_{c}-K_{s}
$$


where $K_{c}$ is the kinetic energy generated by the portions of drops which directly oppose each other and take part in the collision event. $K_{s}$ is the kinetic energy generated by stretching flows due to the portions of drops which do not directly oppose each other. These flows are approximately perpendicular to the reflexive flows. $K_{e}$ is the second source of reflexive action due to the surface-induced flows. When two drops with zero initial velocities are brought into contact, the surface tension effects will force the flow towards the contact point. This phenomenon after the reflexive action will generate opposing flows in the combined mass. These flows are generated by the excess surface energy and the difference between the surface energy of the parent drops and that of the combined mass. The effective reflexive energy $K_{r}^{0}$ can be viewed as the amount of energy that is available for stretching separation.

To extend the model to viscous liquids, we now make the assumption that a fraction $f$ of the effective reflexive separation $K_{r}^{0}$ is dissipated, i.e., the amount of the available effective reflexive energy is $(1-f) K_{r}^{0}$.

Ashgriz and $\mathrm{Poo}^{6}$ postulated that for a nominal spherical combined mass, when the effective kinetic energy is more than $75 \%$ of its nominal surface energy, reflexive separation will occur. This criterion can be explicated in the following relation (with our modification accounting for dissipation):

$$
(1-f) K_{r}^{0} \geq 0.75 \sigma \pi\left(d_{s}^{3}+d_{l}^{3}\right)^{2 / 3} .
$$

The boundary between coalescence and reflexive separation can be derived from Eq. (36) with an equal sign. By comparing the predictions of this model with our direct numerical simulation (DNS) data of the collision outcome, we found (by fitting) the following empirical correlation for $f$ :

$$
f=\frac{5 \mathrm{Ca}}{1+5 \mathrm{Ca}} \text {. }
$$

The model for the boundary between coalescence and reflexive separation is expressed then as

$$
\begin{aligned}
W e= & \frac{12 \Delta\left(1+\Delta^{3}\right)^{2}}{\Delta^{6} \eta_{s}+\eta_{l}} \\
& \frac{\left[(1.75-f)\left(1+\Delta^{3}\right)^{\frac{2}{3}}-(1-f)\left(1+\Delta^{2}\right)\right]}{1-f} .
\end{aligned}
$$

Here $\eta_{s}$ and $\eta_{l}$ are the geometric factors introduced by Ashgriz and Poo, ${ }^{6}$ see Eqs. (11) and (12).

\section{Collision outcome regime maps}

Figure 10 shows the collision outcome regime maps for constant Cas 0.1, 0.5, and 1. The proposed model for the boundaries between coalescence-stretching separation, Eq. (32), and coalescence-reflexive separation, Eq. (38), is shown as black and green lines. The Ashgriz and $\mathrm{Poo}^{6}$ models for water are also provided with the purpose of showing the influence of the viscosity on the collision outcomes. Moreover, we show the Jiang, Umemura, and $\mathrm{Law}^{21}$ prediction for the coalescence-stretching separation boundary, which also includes the viscosity effects. Moving from $\mathrm{Ca}$ 0.1 to 1 , the transitions between coalescence and both types of separation shift to a higher We. This trend confirms the general experimental observation of Kuschel and Sommerfeld. ${ }^{28}$ Note that our empirically derived model describes the boundaries more accurately than both aforementioned existing models.
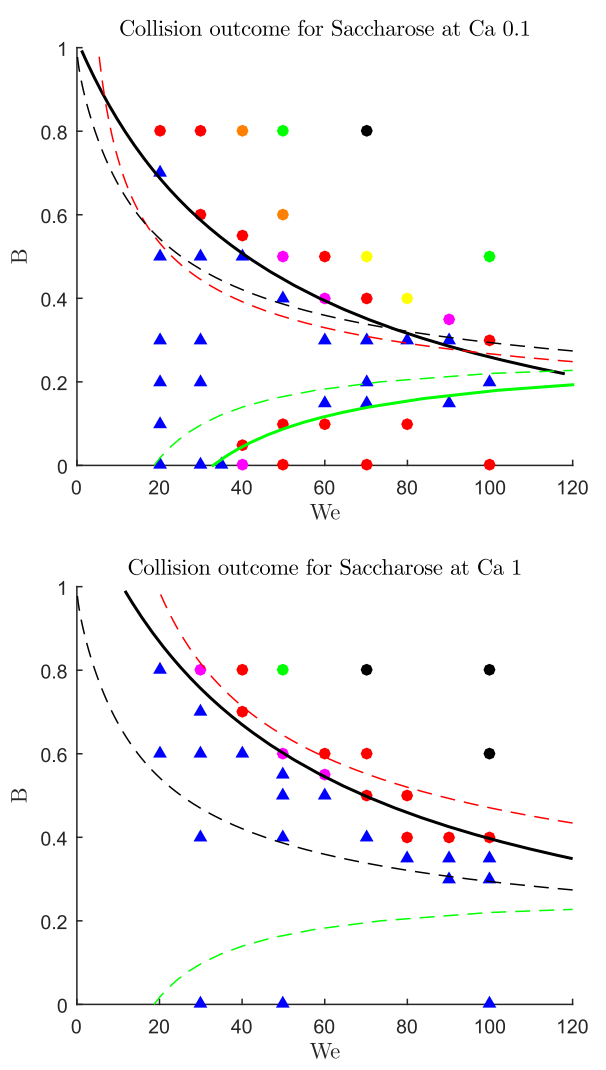
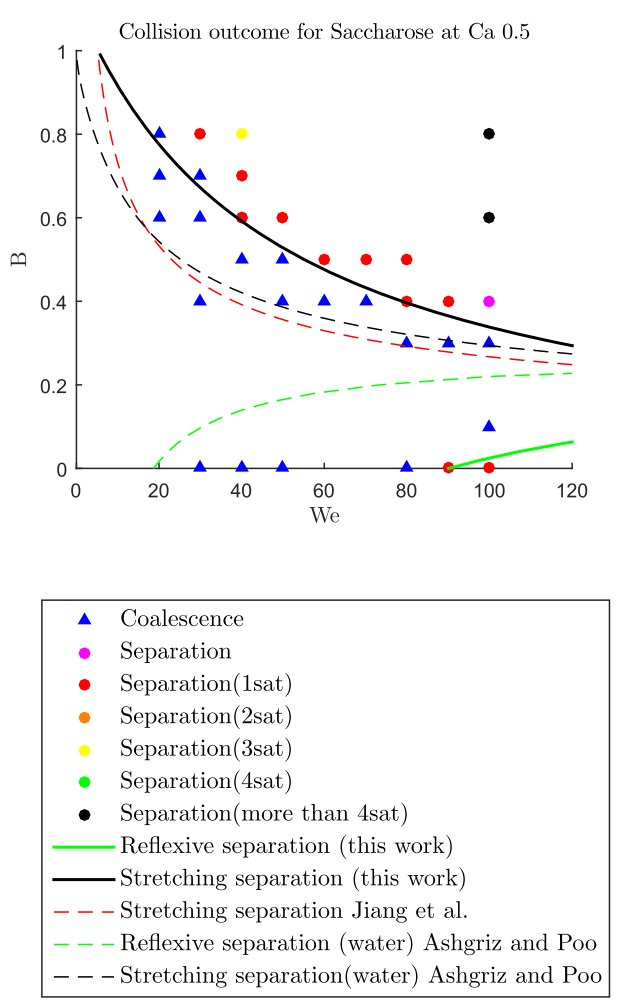

FIG. 10. Collision outcomes regimes for $\mathrm{Ca} 0.1,0.5$, and 1 with coalescencestretching separation and coalescencereflexive separation boundary models. 
The models for stretching separation and reflexive separation, expressed by We, B, $\Delta$, and $\mathrm{Ca}$ [Fig. 11(a)], can also be expressed as a function of We, B, $\Delta$, and Oh [Fig. 11(b)]. The Oh values used in Fig. 11(b) are derived from the averages of the different $\mathrm{Oh}$ values corresponding to a We at a constant Ca. For example, for $\mathrm{Ca} 0.5$, we have $\mathrm{Oh}=0.11$ at $\mathrm{We}=20$ and $\mathrm{Oh}=0.045$ at $\mathrm{We}=100$, so we considered $\mathrm{Oh}=0.075$ to represent the model. The reflexive separation model at $\mathrm{Oh}$ $=0.15$ does not appear in Fig. 11(b) because the critical We at $B=0$ is equal to 310 . Figure 11 shows that for both boundary lines, when moving from a lower to higher $\mathrm{Ca}$ or $\mathrm{Oh}$, the boundaries shift towards a higher We promoting coalescence as the collision outcome. The expression of the coalescencestretching separation model, for $\Delta=1$, as a function of the $\mathrm{Oh}$ is

$$
B=\frac{a_{3}-\frac{W e}{W e+48}}{a_{3} \frac{1}{1+\frac{1}{4} O h \sqrt{W e}}},
$$

Collision outcome boundary models function of $\mathrm{Ca}$

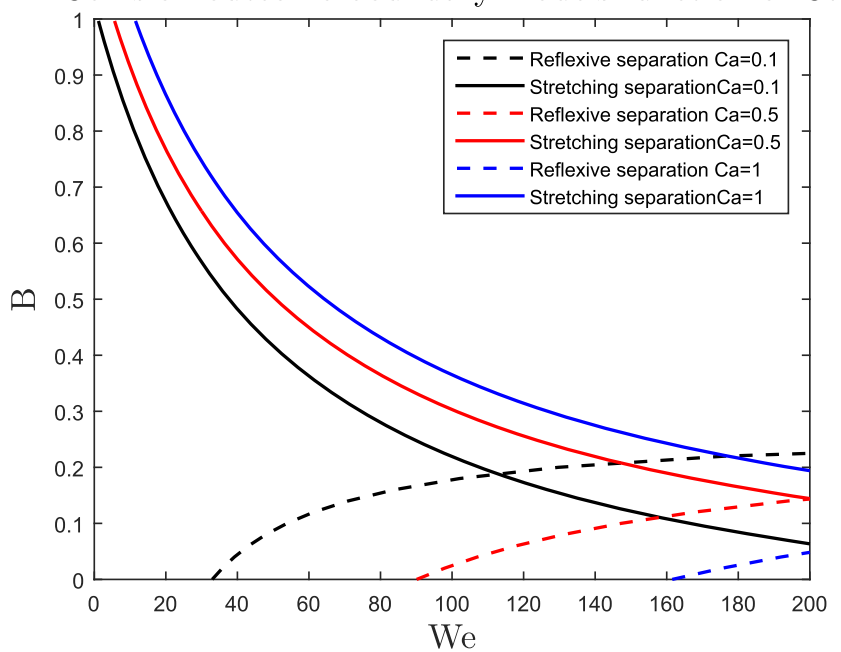

(a)

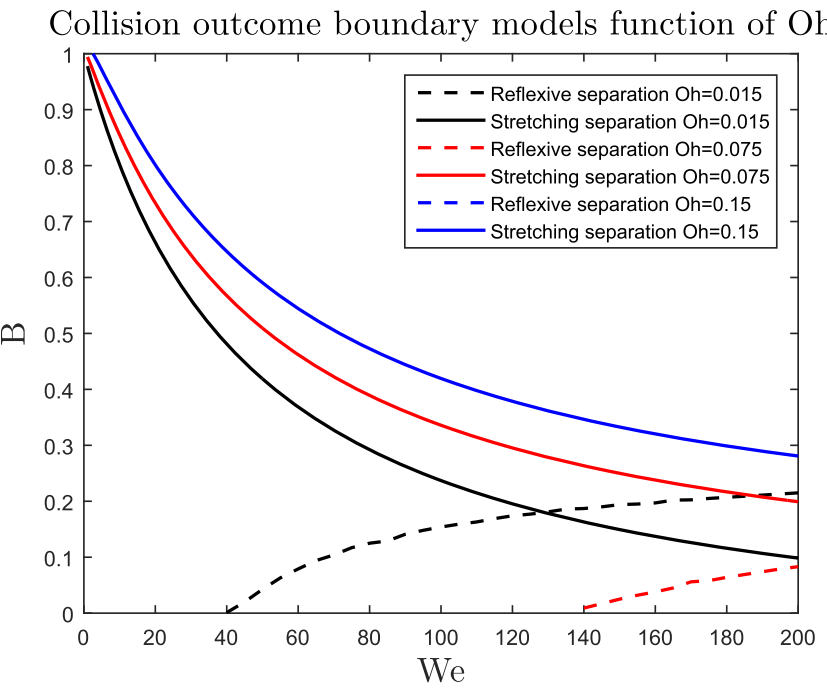

(b)

FIG. 11. Collision outcomes models (a) for the constant capillary number, Eqs. (32) and (38), and (b) for the constant Ohnesorge number, Eqs. (39) and (41).

$$
a_{3}=\frac{21}{22}(\mathrm{Oh} \sqrt{W e})^{1 / 22} .
$$

For the coalescence-reflexive separation model a numerical solution was found for different Wes due to the complexity of the model correlation

$$
\begin{aligned}
2\left[2(1-B)^{2}\left(1-B^{2}\right)\right] & =\frac{48}{W e} \frac{(1.75-f) 2^{2 / 3}-2(1-f)}{1-f}, \\
f & =\frac{5 O h \sqrt{W e}}{1+5 O h \sqrt{W e}} .
\end{aligned}
$$

\section{v. CONCLUSIONS}

The effect of viscosity on binary equal-sized droplet collision dynamic is investigated for a wide range of impacting conditions. Weber numbers ranging from 20 to 100, impact parameters B from 0 to 0.8 , and capillary numbers from 0.1 to 1 were considered for our DNS study. The collision outcomes of coalescence, stretching, and reflexive separation were captured while the model does not allow a study of the bouncing regime. In general, the wide range of parameters introduces a high complexity in the evolution of the interphase topology and for each case a different profile of the viscous dissipation rate during the collision process. Nevertheless, we were able to summarize our observations in relatively simple correlations.

The transition between coalescence and stretching separation was derived through an analysis of the viscous dissipation relative to the total energy budget during the collision event. The model, expressed in terms of the $\mathrm{We}, \mathrm{B}, \Delta$, and Ca, enables a considerable and satisfactory prediction of the influence of droplet viscosity on the boundary.

The coalescence-reflexive separation boundary is visible only in the $\mathrm{Ca} 0.1$ and partially in the $\mathrm{Ca} 0.5$ regime maps so that a complete energy analysis was not possible. A model based on the Ashgriz and $\mathrm{Poo}^{6}$ theory for water was extended to include the viscous effects. Although the empirical model is crude, including a single parameter $f$ (the dissipated fraction of the effective reflexive energy), it has the benefit of predicting not only the onset of reflexive separation at $B=0$ but also the complete boundary for $B>0$. Due to computational limitations, this study focused on a range of capillary numbers $(\mathrm{Ca}=0.1-1)$ where most changes from a non-viscous to a viscous liquid are expected to take place. Moreover, we focused on equal sized droplets $(\Delta=1)$. The empirical correlations are therefore only strictly valid in this range of parameter and should be checked outside this range in future work.

\section{ACKNOWLEDGMENTS}

The authors gratefully acknowledge the financial support of this work by Tetra Pak, Heerenveen. This work was carried out on the Dutch national e-infrastructure with the support of SURF Cooperative.

\footnotetext{
${ }^{1}$ Y. Pan and K. Suga, "Numerical simulation of binary liquid droplet collision," Phys. Fluids 17, 082105 (2005).

${ }^{2}$ M. Nobari, Y.-J. Jan, and G. Tryggvason, "Head-on collision of drops-a numerical investigation," Phys. Fluids 8, 29-42 (1996).
} 
${ }^{3}$ N. Nikolopoulos, K.-S. Nikas, and G. Bergeles, "A numerical investigation of central binary collision of droplets," Comput. Fluids 38, 1191-1202 (2009).

${ }^{4}$ J. Adam, N. Lindblad, and C. Hendricks, "The collision, coalescence, and disruption of water droplets," J. Appl. Phys. 39, 5173-5180 (1968).

${ }^{5}$ V. Arkhipov, G. Ratanov, and V. Trofimov, "Experimental investigation of the interaction of colliding droplets," J. Appl. Mech. Tech. Phys. 19, 201-204 (1978).

${ }^{6}$ N. Ashgriz and J. Poo, "Coalescence and separation in binary collisions of liquid drops,” J. Fluid Mech. 221, 183-204 (1990).

${ }^{7}$ J. Qian and C. Law, "Regimes of coalescence and separation in droplet collision,” J. Fluid Mech. 331, 59-80 (1997).

${ }^{8}$ S. O. Unverdi and G. Tryggvason, "A front-tracking method for viscous, incompressible, multi-fluid flows,” J. Comput. Phys. 100, 25-37 (1992).

${ }^{9}$ M. Nobari and G. Tryggvason, "Numerical simulations of three-dimensional drop collisions," AIAA J. 34, 750-755 (1996).

${ }^{10}$ J.-P. Estrade, H. Carentz, G. Lavergne, and Y. Biscos, "Experimental investigation of dynamic binary collision of ethanol droplets-a model for droplet coalescence and bouncing," Int. J. Heat Fluid Flow 20, 486-491 (1999).

${ }^{11}$ B. Sakakibara and T. Inamuro, "Lattice boltzmann simulation of collision dynamics of two unequal-size droplets," Int. J. Heat Mass Transfer 51, 3207-3216 (2008).

${ }^{12}$ T. L. Georjon and R. D. Reitz, "A drop-shattering collision model for multidimensional spray computations," Atomization Sprays 9, 231 (1999).

${ }^{13}$ A. Munnannur and R. D. Reitz, "A new predictive model for fragmenting and non-fragmenting binary droplet collisions," Int. J. Multiphase Flow 33, 873-896 (2007).

${ }^{14}$ M. Passandideh-Fard and E. Roohi, "Coalescence collision of two droplets: Bubble entrapment and the effects of important parameters," in 14th Annual (International) Mechanical Engineering Conference, Isfahan, 2006.

${ }^{15}$ N. Nikolopoulos, A. Theodorakakos, and G. Bergeles, "Off-centre binary collision of droplets: A numerical investigation,” Int. J. Heat Mass Transfer 52, 4160-4174 (2009).

${ }^{16}$ M. Dai and D. P. Schmidt, "Numerical simulation of head-on droplet collision: Effect of viscosity on maximum deformation," Phys. Fluids 17, 041701 (2005).

${ }^{17}$ X. Chen, D. Ma, P. Khare, and V. Yang, "Energy and mass transfer during binary droplet collision," in Proceedings of 49th AIAA Aerospace Sciences Meeting Including the New Horizons Forum and Aerospace Exposition, 2011.

${ }^{18}$ C. Planchette, H. Hinterbichler, M. Liu, D. Bothe, and G. Brenn, "Colliding drops as coalescing and fragmenting liquid springs," J. Fluid Mech. 814, 277-300 (2017).

${ }^{19} \mathrm{G}$. Brenn and A. Frohn, "Collision and merging of two equal droplets of propanol," Exp. Fluids 7, 441-446 (1989).

${ }^{20}$ N. Ashgriz and P. Givi, "Coalescence efficiencies of fuel droplets in binary collisions," Int. Commun. Heat Mass Transfer 16, 11-20 (1989).

${ }^{21}$ Y. Jiang, A. Umemura, and C. Law, "An experimental investigation on the collision behaviour of hydrocarbon droplets," J. Fluid Mech. 234, 171-190 (1992).
${ }^{22} \mathrm{~K}$. Willis and M. Orme, "Experiments on the dynamics of droplet collisions in a vacuum," Exp. Fluids 29, 347-358 (2000).

${ }^{23} \mathrm{~K}$. Willis and M. Orme, "Binary droplet collisions in a vacuum environment: An experimental investigation of the role of viscosity," Exp. Fluids 34, 28-41 (2003).

${ }^{24} \mathrm{G}$. Brenn, D. Valkovska, and K. Danov, "The formation of satellite droplets by unstable binary drop collisions," Phys. Fluids 13, 2463-2477 (2001).

${ }^{25} \mathrm{G}$. Brenn and V. Kolobaric, "Satellite droplet formation by unstable binary drop collisions," Phys. Fluids 18, 087101 (2006).

${ }^{26}$ C. Gotaas, P. Havelka, H. A. Jakobsen, H. F. Svendsen, M. Hase, N. Roth, and B. Weigand, "Effect of viscosity on droplet-droplet collision outcome: Experimental study and numerical comparison,” Phys. Fluids 19, 102106 (2007).

${ }^{27}$ O. Kurt, U. Fritsching, and G. Schulte, "Binary collisions of droplets with fluid and suspension particles," ILASS 2007, 10-12.

${ }^{28}$ M. Kuschel and M. Sommerfeld, "Investigation of droplet collisions for solutions with different solids content," Exp. Fluids 54, 1-17 (2013).

${ }^{29} \mathrm{M}$. Sommerfeld and M. Kuschel, "Modelling droplet collision outcomes for different substances and viscosities," Exp. Fluids 57, 187 (2016).

${ }^{30} \mathrm{~K}$. Krishnan and E. Loth, "Effects of gas and droplet characteristics on drop-drop collision outcome regimes, ’ Int. J. Multiphase Flow 77, 171-186 (2015).

${ }^{31}$ G. H. Ko and H. S. Ryou, "Modeling of droplet collision-induced breakup process," Int. J. Multiphase Flow 31, 723-738 (2005).

${ }^{32}$ M. Van Sint Annaland, N. Deen, and J. Kuipers, "Numerical simulation of gas bubbles behaviour using a three-dimensional volume of fluid method," Chem. Eng. Sci. 60, 2999-3011 (2005).

${ }^{33} \mathrm{M}$. Baltussen, J. Kuipers, and N. Deen, "A critical comparison of surface tension models for the volume of fluid method," Chem. Eng. Sci. 109, 65-74 (2014).

${ }^{34}$ D. L. Youngs, "Time-dependent multi-material flow with large fluid distortion," in Numerical Methods for Fluid Dynamics, edited by K. W. Morton and M. J. Baines (Academic, New York, 1982).

${ }^{35}$ G. Tryggvason, B. Bunner, A. Esmaeeli, D. Juric, N. Al-Rawahi, W. Tauber, J. Han, S. Nas, and Y.-J. Jan, "A front-tracking method for the computations of multiphase flow,” J. Comput. Phys. 169, 708-759 (2001).

${ }^{36}$ W. Dijkhuizen, I. Roghair, M. V. S. Annaland, and J. Kuipers, "DNS of gas bubbles behaviour using an improved 3D front tracking model-Model development," Chem. Eng. Sci. 65, 1427-1437 (2010).

${ }^{37}$ K.-L. Pan, C. K. Law, and B. Zhou, "Experimental and mechanistic description of merging and bouncing in head-on binary droplet collision," J. Appl. Phys. 103, 064901 (2008).

${ }^{38}$ G. MacKay and S. Mason, "The gravity approach and coalescence of fluid drops at liquid interfaces,” Can. J. Chem. Eng. 41, 203-212 (1963).

${ }^{39}$ M. Kwakkel, W.-P. Breugem, and B. J. Boersma, "Extension of a clsvof method for droplet-laden flows with a coalescence/breakup model," J. Comput. Phys. 253, 166-188 (2013).

${ }^{40}$ J. T. Padding, N. G. Deen, E. F. Peters, and J. H. Kuipers, "Euler-Lagrange modeling of the hydrodynamics of dense multiphase flows," Adv. Chem. Eng. 46, 137-191 (2015), Chap. 3. 